\title{
The curve shortening problem under Robin boundary condition
}

\author{
Kai-Seng Chou and Xiao-Liu Wang
}

\begin{abstract}
The curve shortening problem for a graph under Robin boundary condition is studied in this paper. The large time behavior of the global solution is shown to depend critically on the parameters in the boundary condition. Some asymptotic behavior of the solution is also discussed.
\end{abstract}

Mathematics Subject Classification (2010). Primary 53C44;

Secondary 35B05, 35B30, 35B50.

Keywords. Curve shortening problem, Long-time behavior of solution, Robin boundary condition.

\section{Introduction}

In the curve shortening problem one studies the motion of a curve driven along its normal direction by its curvature. Since the pioneering works of GageHamilton [10] and Grayson [12], there have been many results for this problem and one may consult the books Cao [6], Chou-Zhu [9], Giga [11] and Zhu [23].

When the curve is expressible as the graph of a function, the curve shortening problem can be reformulated as the following quasilinear parabolic equation

$$
u_{t}=\frac{u_{x x}}{1+u_{x}^{2}}, \quad t>0,
$$

where the curve at time $t$ is given by the graph of $u(., t)$. The boundary value problem for this equation was studied in Huisken [13] together with the mean curvature flow. For the Dirichlet problem it is shown that any global solution of (1.1) converges to the linear function satisfying the boundary values at the two endpoints, and for the homogeneous Neumann problem, the solution converges to a constant as time goes to infinity. The non-homogeneous Neumann problem was subsequently studied in Altschuler-Wu [1] where the asymptotic behavior of the solution is determined (see below). One may consult [19] for 


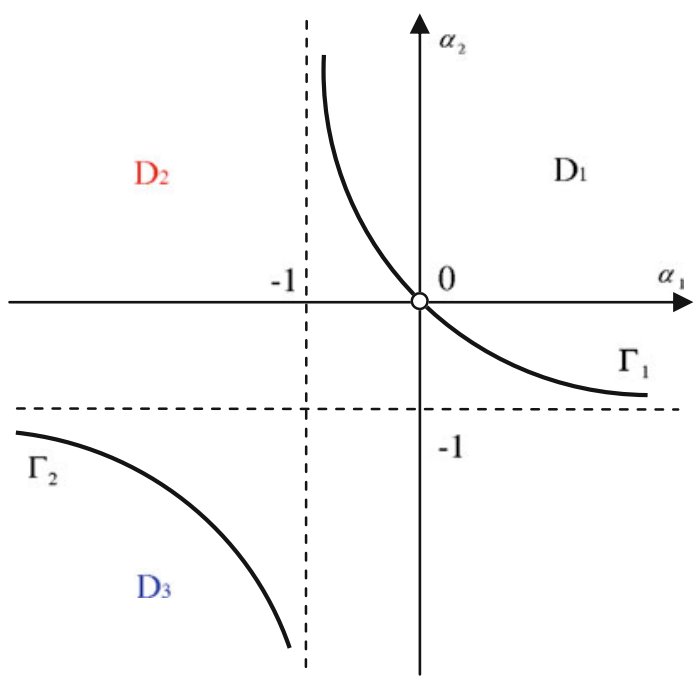

Figure 1. The division of $\left(\alpha_{1}, \alpha_{2}\right)$.

results on other boundary value problems for the curve shortening problems. In connection to its applications in image analysis [20], the curve shortening problem is regarded as the geometric invariant replacement of the heat equation. In view of its potential applications in other areas, it is desirable to undertake a thorough investigation of (1), where results already obtained for the heat equation may be raised as questions for the curve shortening problem. Among many well-studied boundary conditions, the Robin condition is next common to the Dirichlet and Neumann conditions. It is the purpose of this paper to study $(1)$ in $[0,1] \times(0, \infty)$ under Robin boundary condition

$$
\begin{aligned}
& u_{x}(0, t)=\alpha_{1} u(0, t)+\beta_{1}, \\
& u_{x}(1, t)=-\alpha_{2} u(1, t)+\beta_{2},
\end{aligned}
$$

where $\alpha=\left(\alpha_{1}, \alpha_{2}\right) \neq(0,0)$ and $\beta=\left(\beta_{1}, \beta_{2}\right)$ are given. One can show that for any smooth initial function satisfying (1.2) problem (1.1) under (1.2) has a global solution, see Sect. 1. Our goal is to study the large time behavior of the global solution. It turns out that it depends critically on the parameters $\alpha$ and $\beta$. To formulate it, we divide $\mathbb{R}^{2} \backslash\{(0,0)\}$ into the disjoint union of the following sets: $D_{1}=\left\{\alpha: \delta>0, \alpha_{1}\right.$ or $\left.\alpha_{2}>0\right\}, D_{2}=\{\alpha: \delta<0\}, D_{3}=\{\alpha: \delta>$ $\left.0, \alpha_{1}, \alpha_{2}<0\right\}, \Gamma_{1}=\left\{\alpha: \delta=0, \alpha_{1} \alpha_{2}<0\right\}$, and $\Gamma_{2}=\left\{\alpha: \delta=0, \alpha_{1}, \alpha_{2}<0\right\}$, where $\delta=\alpha_{1}+\alpha_{2}+\alpha_{1} \alpha_{2}$. See Fig. 1 .

This division is partly due to the steady states for the problem. It is clear that any steady state of (1.1) is a linear function. When $\delta$ is non-zero, there is always a unique linear function satisfying (1.2), hence the steady state is unique. On the other hand, when $\delta=0$, steady states exist if and only if the compatibility condition: $\beta_{2}-\left(1+\alpha_{2}\right) \beta_{1}=0$, that's, $\alpha_{1} \beta_{2}+\alpha_{2} \beta_{1}=0$, holds. When this happens, there are infinitely many steady states satisfying (1.2). 
It is not hard to show that there are only four types of possible large time behavior (see Sect. 2) for any global solution of (1.1) under (1.2):

Case 1. The solution converges uniformly to a steady state.

Case 2. The solution diverges to infinity uniformly, that's, $\min u(., t)$ tends to $\infty$ as $t$ tends to $\infty$.

Case 3. The solution diverges to negative infinity uniformly, that's, max $u(., t)$ tends to $-\infty$ as $t$ tends to $\infty$.

Case 4. The solution becomes unbounded from both sides, that is, $\max u(., t)$ tends to $\infty$ and $\min u(., t)$ tends to $-\infty$ as $t$ tends to $\infty$.

Our main result shows that the large time behavior becomes more diverse as the parameters $\alpha$ varies from $D_{1}, \Gamma_{1}, D_{2}$, and $\Gamma_{2}$ to $D_{3}$. Specifically we state

Theorem 1.1. Concerning the large time behavior of a global solution of (1.1) under (1.2), we have

(1) For $\alpha \in D_{1}$, only Case 1 holds.

(2) For $\alpha \in \Gamma_{1}$, (a) when $\alpha_{1} \beta_{2}+\alpha_{2} \beta_{1}=0$, only Case 1 holds; (b) when $\alpha_{1} \beta_{2}+\alpha_{2} \beta_{1}<0$, only Case 2 holds; and (c) when $\alpha_{1} \beta_{2}+\alpha_{2} \beta_{1}>0$, only Case 3 holds.

(3) For $\alpha \in D_{2}$, Cases 1 to 3 are possible.

(4) For $\alpha \in \Gamma_{2}$, (a) when $\alpha_{1} \beta_{2}+\alpha_{2} \beta_{1}=0$, Cases 1 to 3 are possible, and (b) when $\alpha_{1} \beta_{2}+\alpha_{2} \beta_{1} \neq 0$, Cases 2 to 4 are possible.

(5) For $\alpha \in D_{3}$, all four cases are possible.

Here a case is possible means that it can be realized by some global solution.

When $\alpha_{1}$ and $\alpha_{2}$ are zero, problem (1.1) under (1.2) becomes Neumann problem and is discussed in [1], where it is shown that for any global solution only Case 1 holds when $\beta_{1}=\beta_{2}$, only Case 2 holds when $\beta_{2}>\beta_{1}$, and only Case 3 holds when $\beta_{2}<\beta_{1}$.

Naturally one is led to the study of the asymptotic behavior of the unbounded solutions. Here we give some preliminary results for those solutions whose slopes at endpoints become very large and are of the same sign eventually. We formulate them in two propositions.

Proposition 1.2. Let $\alpha$ be in $\Gamma_{1} \cup\left(D_{2} \cap\left\{\alpha: \alpha_{1} \alpha_{2}<0\right\}\right)$. For any convex (resp. concave) solution $u$ of (1.1) under (1.2), if $\min _{x} u(x, t) \rightarrow \infty$ (resp. $\left.\max _{x} u(x, t) \rightarrow-\infty\right)$ as $t \rightarrow \infty$, then for any $\rho \in(0,1 / 2)$, the curvature of the graph of $u(., t)$ inside the set $[\rho, 1-\rho] \times \mathbb{R}$ tends to zero uniformly as $t$ tends to $\infty$.

Proposition 1.3. Let $\alpha$ be in $\Gamma_{2} \cup D_{3}$. Assume that $u$ is a solution of (1.1) under (1.2) which is unbounded from both sides. Then for any $c>0$, the curvature of the graph of $u(., t)$ inside the set $[0,1] \times[-c, c]$ tends to zero uniformly as $t$ tends to $\infty$.

We leave out the asymptotic behavior of those solutions with $\alpha$ belonging to $D_{2} \cap\left\{\alpha: \alpha_{1} \alpha_{2} \geq 0\right\}, \Gamma_{2}$ or $D_{3}$ which diverge to infinity or negative infinity uniformly. For Neumann problem, it is known that any unbounded solution is 
asymptotic to a portion of a grim reaper [1]. By analogous thinking we conjecture that in this case, the solution is asymptotic to the grim reaper over $(0,1)$ when its slopes go to infinity at both endpoints, or to a portion of a grim reaper when its slope tends to infinity only at one endpoint.

For related works, we point out the papers of Kohsaka [15] and ChernGuo-Lo [7] on free boundary problems for (1.1), and Altschuler-Wu [2], Stahl [21] and Huisken [14] for the mean curvature flow under a geometric Neumann condition.

\section{Global solutions}

Proposition 2.1. Let $u_{0}$ be a function in $C^{2, \sigma}([0,1])$ for some $\sigma$ in $(0,1)$ satisfying (1.2). The problem (1.1) under (1.2) has a unique global solution $u$ satisfying $u(., 0)=u_{0}$ which belongs to the parabolic Hölder space $\tilde{C}^{2, \sigma}([0,1] \times$ $[0, T])$ for every $T>0$.

Proof. According to Theorem 8.9 in Lieberman [17] the existence of a solution $u$ of $(1.1)$ and $(1.2)$ in $[0, T]$ follows from an a priori estimate of the form

$$
\|u\|_{\tilde{C}^{2, \sigma}} \leq C\left(\alpha, \beta, T,\left\|u_{0}\right\|_{C^{2, \sigma}}\right)
$$

where $\alpha=\left(\alpha_{1}, \alpha_{2}\right)$ and $\beta=\left(\beta_{1}, \beta_{2}\right)$ are specified in (1.2). From the structure of (1.1), clearly it suffices to derive a weaker estimate, namely,

$$
\|u\|_{\tilde{C}^{1, \sigma^{\prime}}} \leq C\left(\alpha, \beta, T,\left\|u_{0}\right\|_{C^{2, \sigma}}\right)
$$

for some $\sigma^{\prime} \in(0,1)$. Then the desired second order estimate follows from Schauder estimate for linear parabolic equations.

We proceed to derive (2.1). In the following $u$ is a $\tilde{C}^{2, \sigma}$-solution of (1.1) under $(1.2)$ in $[0,1] \times[0, T]$.

Step 1 (Uniform estimate) We note that (1.1) admits a traveling wave solution called the grim reaper

$$
\begin{aligned}
& g(x, t)=\frac{1}{c} \log \sec \left(c\left(x-\frac{1}{2}\right)\right)+c t+d, \\
& (x, t) \in\left(\frac{1}{2}-\frac{\pi}{2 c}, \frac{1}{2}+\frac{\pi}{2 c}\right) \times(-\infty, \infty),
\end{aligned}
$$

where $c \in(0, \pi)$ and $d \in \mathbb{R}$ are two parameters. We observe that $g$ is welldefined on $[0,1]$ and blows up to $\infty$ at $1 / 2 \pm \pi / 2 c$. When $c$ is very closed to $\pi$, the graph of $g$ becomes very steep at 0 and 1 .

We shall choose $d$ and $c$ so that $g(x, t) \geq u(x, t)$ for $(x, t) \in[0,1] \times[0, T]$. First, fix a large $d$ such that $g(x, 0)>u_{0}(x)$. Next, choose $c$ sufficiently close to $\pi$ such that

$$
\begin{gathered}
g_{x}(0, t)<-\left|\alpha_{1}\right| g(0, t)+\beta_{1}, \\
g_{x}(1, t)>\left|\alpha_{2}\right| g(1, t)+\beta_{2},
\end{gathered}
$$


for all $t$ in $[0, T]$. This is possible because

$$
-g_{x}(0, t)=g_{x}(1, t)=\frac{\sin (c / 2)}{\cos (c / 2)}
$$

tend to $\infty$ as $c$ approaches $\pi$ much faster than

$$
\begin{aligned}
g(0, t)=g(1, t) & =\frac{1}{c} \log \sec \frac{c}{2}+c t+d \\
& \leq-\frac{1}{c} \log \cos \frac{c}{2}+c T+d
\end{aligned}
$$

$(z / \log z \rightarrow \infty$ as $z \rightarrow \infty)$. We claim $g>u$ in $[0, T]$. Clearly this is true at $t=0$. If $g$ touches $u$ at some finite time $t_{1}$, by the strong maximum principle it must occur at $x=0$ or 1 . Let us assume it is at $x=0$. Then, $u\left(0, t_{1}\right)=g\left(0, t_{1}\right)$, and $(g-u)_{x}\left(0, t_{1}\right)>0$. However,

$$
\begin{aligned}
g_{x}\left(0, t_{1}\right) & <-\left|\alpha_{1}\right| g\left(0, t_{1}\right)+\beta_{1} \\
& \leq-\alpha_{1} u\left(0, t_{1}\right)+\beta_{1} \\
& =u_{x}\left(0, t_{1}\right),
\end{aligned}
$$

contradiction holds. We have obtained an upper bound for $u$. A similar lower bound can be obtained by considering a grim reaper with negative $c$.

Step 2 (Gradient estimate) The function $v=u_{x}$ satisfies

$$
v_{t}=\frac{v_{x x}}{1+u_{x}^{2}}-\frac{2 u_{x} u_{x x}}{\left(1+u_{x}^{2}\right)^{2}} v_{x}
$$

and

$$
\begin{aligned}
& v(0, t)=\alpha_{1} u(0, t)+\beta_{1}, \\
& v(1, t)=-\alpha_{2} u(1, t)+\beta_{2} .
\end{aligned}
$$

(Note that by parabolic regularity the solution is in $\tilde{C}^{4, \sigma}$ locally in $(0,1) \times$ $(0, T]$.) Using Step 1 and the maximum principle we conclude

$$
\left\|u_{x}\right\|_{L^{\infty}}=\|v\|_{L^{\infty}} \leq C\left(\alpha, \beta, T,\left\|u_{0}\right\|_{C^{1}}\right) .
$$

Step 3 (Hölder estimate in time) From Step 2 we get

$$
|u(x, t)-u(y, t)| \leq C_{1}|x-y|, \quad x, y \in[0,1], t \in[0, T] .
$$

We shall derive

$$
\left|u\left(x, s_{1}\right)-u\left(x, s_{2}\right)\right| \leq C_{2}\left|s_{1}-s_{2}\right|^{1 / 2}, s_{1}, s_{2} \in[0, T], x \in[0,1] .
$$

$(2.2)$ and (2.3) together imply a Hölder estimate for $u$ for any exponent in $(0,1)$.

We choose two test functions as follows. Let $\xi_{0}$ be a non-negative function on $[0, \infty)$ which equals to 1 near 0 , becomes 0 for all $x \geq 1$ and satisfies $\int_{0}^{1} \xi_{0}(x) d x=1$. We set

$$
\xi(x)=\xi_{0}\left(\frac{\left|x-x_{0}\right|}{\left|s_{2}-s_{1}\right|^{1 / 2}}\right)
$$

where $x_{0} \in[0,1]$ and $0<s_{1}<s_{2} \leq T$ are fixed. For small $\delta_{0}>0$, let

$$
\theta_{\delta_{0}}(t)=\int_{-\infty}^{t} \eta_{\delta_{0}}(\tau) d \tau
$$


where $\eta_{\delta_{0}}(\tau)=\left(-\chi_{\left[s_{1}-\delta_{0} / 2, s_{1}+\delta_{0} / 2\right]}(\tau)+\chi_{\left[s_{2}-\delta_{0} / 2, s_{2}+\delta_{0} / 2\right]}(\tau)\right) / \delta_{0}$. We write (1.1) in the form

$$
u_{t}=\left(\arctan u_{x}\right)_{x} .
$$

Multiplying both sides of this equation by $\xi(x) \theta_{\delta_{0}}(t)$ we have $\int_{0}^{1} \int_{0}^{T} u_{t}(x, t) \xi(x) \theta_{\delta_{0}}(t) d t d x=\int_{0}^{1} \int_{0}^{T}\left(\arctan u_{x}\right)_{x}(x, t) \xi(x) \theta_{\delta_{0}}(t) d t d x$.

Perform integration by parts on the left hand side of this identity to get

$$
\begin{aligned}
\int_{0}^{1} & \int_{0}^{T} u_{t}(x, t) \xi(x) \theta_{\delta_{0}}(t) d t d x=-\int_{0}^{1} \int_{0}^{T} u(x, t) \xi(x) \eta_{\delta_{0}}(t) d t d x \\
= & \frac{1}{\delta_{0}} \int_{0}^{1} \int_{s_{1}-\delta_{0} / 2}^{s_{1}+\delta_{0} / 2} u(x, t) \xi(x) d t d x-\frac{1}{\delta_{0}} \int_{0}^{1} \int_{s_{2}-\delta_{0} / 2}^{s_{2}+\delta_{0} / 2} u(x, t) \xi(x) d t d x \\
& \rightarrow \int_{0}^{1}\left(u\left(x, s_{1}\right)-u\left(x, s_{2}\right)\right) \xi(x) d x
\end{aligned}
$$

as $\delta_{0} \rightarrow 0$. On the other hand,

$$
\begin{aligned}
\int_{0}^{T} & \int_{0}^{1}\left(\arctan u_{x}\right)_{x}(x, t) \xi(x) \theta_{\delta_{0}}(t) d x d t \\
= & -\int_{0}^{T} \int_{0}^{1}\left(\arctan u_{x}\right)(x, t) \xi^{\prime}(x) \theta_{\delta_{0}}(t) d x d t \\
& +\left.\int_{0}^{T}\left(\arctan u_{x}\right)(x, t) \xi(x) \theta_{\delta_{0}}(t)\right|_{0} ^{1} d t
\end{aligned}
$$

Using $|\arctan z| \leq \pi / 2$, and the form of $\xi$, we have

$$
\begin{aligned}
& \left|\int_{0}^{T} \int_{0}^{1}\left(\arctan u_{x}\right)(x, t) \xi^{\prime}(x) \theta_{\delta_{0}}(t) d x d t\right| \\
& \quad \leq \pi|| \xi_{0}^{\prime} \|_{L^{\infty}} \times \frac{1}{\left(s_{2}-s_{1}\right)^{1 / 2}} \times\left(s_{2}-s_{1}\right)^{1 / 2} \times\left|s_{2}-s_{1}+2 \delta_{0}\right| \\
& \quad=\pi|| \xi_{0}^{\prime} \|_{L^{\infty}}\left|s_{2}-s_{1}+2 \delta_{0}\right|,
\end{aligned}
$$

and

$$
\left|\int_{0}^{T}\left(\arctan u_{x}\right)(x, t) \xi(x) \theta_{\delta_{0}}(t)\right|_{0}^{1} d t \mid \leq \frac{\pi}{2}\left(s_{2}-s_{1}+2 \delta_{0}\right) .
$$

Combining with (2.4), we have

$$
\left|\int_{0}^{1}\left(u\left(x, s_{1}\right)-u\left(x, s_{2}\right)\right) \xi(x) d x\right| \leq C\left|s_{1}-s_{2}\right| .
$$

Now,

$$
\begin{aligned}
\left|u\left(x_{0}, s_{1}\right)-u\left(x_{0}, s_{2}\right)\right| \leq & \left|u\left(x_{0}, s_{1}\right)-u\left(x, s_{1}\right)\right|+\left|u\left(x, s_{1}\right)-u\left(x, s_{2}\right)\right| \\
& +\left|u\left(x, s_{2}\right)-u\left(x_{0}, s_{2}\right)\right| \\
\leq & 2 C_{1}\left|x-x_{0}\right|+\left|u\left(x, s_{1}\right)-u\left(x, s_{2}\right)\right| .
\end{aligned}
$$


Therefore,

$$
\begin{aligned}
& \left|u\left(x_{0}, s_{1}\right)-u\left(x_{0}, s_{2}\right)\right|\left|s_{1}-s_{2}\right|^{1 / 2} \leq \int_{0}^{1}\left|u\left(x_{0}, s_{1}\right)-u\left(x_{0}, s_{2}\right)\right| \xi(x) d x \\
& \quad \leq 2 C_{1} \int_{0}^{1}\left|x-x_{0}\right| \xi(x) d x+\int_{0}^{1}\left|u\left(x, s_{1}\right)-u\left(x, s_{2}\right)\right| \xi(x) d x \\
& \quad \leq C\left|s_{1}-s_{2}\right|
\end{aligned}
$$

after using (2.5), and (2.3) follows.

Step 4 (Gradient Hölder estimate) The function $v=u_{x}$ satisfies

$$
v_{t}=\left(\frac{v_{x}}{1+v^{2}}\right)_{x},
$$

and

$$
\begin{aligned}
& v(0, t)=\alpha_{1} u(0, t)+\beta_{1}, \\
& v(1, t)=-\alpha_{2} u(1, t)+\beta_{2} .
\end{aligned}
$$

This equation can be viewed as a linear parabolic equation in divergence form with a bounded coefficient $\left(1+v^{2}\right)^{-1}$ whose solution $v$ satisfies a Hölder condition at the boundary from Step 3. Using standard regularity theory [LSU] we conclude that $v$ belongs to $\widetilde{C}^{\sigma^{\prime}}([0,1] \times[0, T])$ for some $\sigma^{\prime} \in(0,1)$, so $(2.1)$ holds.

\section{Some lemmas}

Lemma 3.1. Any solution $u$ of (1.1) under (1.2) satisfies $u_{t}>0$ (resp. $\left.<0\right)$ for all $t \geq 0$ if $u_{t}(x, 0)>0($ resp. $<0)$ on $[0,1]$.

Proof. By differentiating (1.1) and (1.2) in time, we see that $u_{t}$ satisfies a linear parabolic equation together with the boundary condition:

$$
\begin{aligned}
& \left(u_{t}\right)_{x}(0, t)=\alpha_{1} u_{t}(0, t), \\
& \left(u_{t}\right)_{x}(1, t)=-\alpha_{2} u_{t}(1, t) .
\end{aligned}
$$

Now suppose there is a first time $t_{1}>0$ such that $u_{t}=0$. By the strong maximum principle, min $u_{t}\left(x, t_{1}\right)$ attains at $x=0$ or $x=1$, so $u_{t}\left(0, t_{1}\right)=0$ or $u_{t}\left(1, t_{1}\right)=0$. However, by the parabolic Hopf's lemma we have $u_{t x}\left(0, t_{1}\right)>0$ or $u_{t x}\left(1, t_{1}\right)<0$, contradicting $(3.1)$. So $u_{t}$ must remain positive or negative all time.

Lemma 3.2. For any global solution $u$ of (1.1) under (1.2), there exists some $t_{0} \geq 0$ such that $u_{t}(x, t)$ does not change sign at $x=0$ and 1 for all $t \geq t_{0}$.

Proof. See Lemma 1 in Brunovský, et al [5].

Lemma 3.3. Any global unbounded solution u must fall into one of the following cases: (a) $\min _{x} u(x, t) \rightarrow \infty$, (b) $\max _{x} u(x, t) \rightarrow-\infty$, and (c) $\max _{x} u(x, t) \rightarrow \infty$ and $\min _{x} u(x, t) \rightarrow-\infty$. 
Proof. Let $u$ be a global unbounded solution of (1.1) under (1.2). By replacing $u$ by $-u$ if necessary, we may assume without loss of generality that $\max u(x, t) \rightarrow \infty$ as $t \rightarrow \infty$. By the strong maximum principle, the maximum of $u(x, t)$ cannot attain in the interior of $[0,1]$, so it attains either at $x=0$ or $x=1$. If it is the latter case, we may replace $u(x)$ by $u(1-x)$, hence it suffices to assume $u(0, t) \rightarrow \infty$ by Lemma 3.2. By the same lemma, if $u$ does not tend to $\infty$ or $-\infty$ at $x=1$, we can find a number $M$ such that $M \leq u(1, t) \leq M+1$ for all large time. Then

$$
u_{x}(1, t)=-\alpha_{2} u(1, t)+\beta_{2} \geq-M^{\prime}
$$

is bounded for all large $t$. Now, fix a grim reaper $g_{0}(., 0)$ over $[0,1]$ whose slope at $x=1$ is less than $-M^{\prime}$ and blows up at $x=-1$, say. Let $L=g_{0}(0,0)-$ $g_{0}(1,0)$. As $u(0, t) \rightarrow \infty$, we can find a $T$ such that $u(0, t)>L+|M|+1$ for all $t \geq T$. Let $g_{1}=g_{0}-d$ where $d$ is a large number so that $u(x, T)>g_{1}(x)$ on $[0,1]$. Then by the comparison principle $u(\cdot, t)$ and $g_{1}(\cdot, t)$ cannot touch at $x=0$ and 1 and so $u(x, t)>g_{1}(x, t)$ for all $t \geq T$. Contradiction holds because $\min _{x} g_{1}(x, t) \rightarrow \infty$. We conclude that $\min u(1, t)$ must tend to $\infty$ or $-\infty$ as $t \rightarrow \infty$.

\section{Large time behavior}

In this section we prove Theorem 1.1. We shall need the following auxiliary functions. First, let

$$
w(x)=a+\left(\alpha_{1} a+\beta_{1}\right) x+\left(\frac{-\delta a+\beta_{2}-\beta_{1}-\alpha_{2} \beta_{1}}{2 m+\alpha_{2}}\right) x^{2 m},
$$

where $m$ is a positive number chosen so that both $2 m-2$ and $2 m+\alpha_{2}$ are positive and $w(0)=a$ is a free parameter to be specified later. For each fixed $a, w$ satisfies the Robin boundary condition (1.2). Next, let

$$
l(x)=a\left(1+\alpha_{1} x\right)+\beta_{1} x .
$$

This linear function satisfies half of (1.2) at $x=0$. It also satisfies (1.2) at $x=1$ (and hence is a steady state) if and only if $\delta a=\beta_{2}-\left(1+\alpha_{2}\right) \beta_{1}$, where $\delta=\alpha_{1}+\alpha_{2}+\alpha_{1} \alpha_{2}$. Similarly, the linear function

$$
\tilde{l}(x)=a\left[1+\alpha_{2}(1-x)\right]+\beta_{2}(x-1)
$$

satisfies half of (1.2) at $x=1$.

Now, we start the proof of Theorem 1.1.

First, consider $\alpha \in D_{1}$. In this case the problem has a unique steady state $l_{s}$. We claim that any global solution $u$ will converge to $l_{s}$ uniformly as $t$ tends to infinity. To this end it suffices to show every global solution is bounded uniformly in time, for then the general theorem of Zelenjak [22] yields the desired conclusion.

To show a uniform bound for $u$ we make use of the function $w$ in (4.1). As $\delta$ is positive, we see immediately from (4.1) that $w$ is a concave (resp. convex) function with $\min _{x} w(x) \rightarrow \infty\left(\max _{x} w(x) \rightarrow-\infty\right)$ as $a \rightarrow \infty($ resp. $a \rightarrow-\infty)$. 
Therefore, for any initial function $u_{0}$ we can fix a large $a$ so that $w_{-|a|}<u_{0}<$ $w_{a}$. It follows from Lemma 3.1 and the comparison principle that $u$ is bounded between $w_{-|a|}$ and $w_{a}$ for all time.

Second, consider $\alpha$ in $\Gamma_{1}$. Let $\gamma \equiv \beta_{2}-\left(1+\alpha_{2}\right) \beta_{1}=\left(\alpha_{1} \beta_{2}-\alpha_{2} \beta_{1}\right) / \alpha_{1}$. When $\gamma$ is equal to zero, this problem has infinitely many steady states given by (4.2). As $|a| \rightarrow \infty$, the zero of $l$ approaches the number $-1 / \alpha_{1}$, which does not belong to $(0,1)$ (noting that $\left.\alpha_{1}+1>0\right)$. Therefore, for large $a, l(x) \rightarrow \infty$ (resp. $-\infty$ ) uniformly as $a \rightarrow \infty$ (resp. $-\infty$ ). It follows that for any initial $u_{0}$, we can find a large $a$ such that $l_{-|a|}<u_{0}<l_{a}$. By Lemma 3.1 and the comparison principle again, the solution $u$ is pinched between $l_{a}$ and $l_{-|a|}$ for all time, and we conclude the uniform convergence as in the previous case.

When $\gamma$ is not equal to zero, we consider the case where $\alpha_{1}<0$ and $\alpha_{2}>0$ first. For $\gamma>0$, we claim that all global solutions diverge to $\infty$ as $t \rightarrow \infty$. To see this, take $l$ in (4.2) with a very negative $a$ so that $l<u_{0}$ in $[0,1]$. We claim that $l(x)<u(x, t)$ for all $t$. For, if $u$ touches $l$ at some first time $t_{1}$, by the strong maximum principle it must touch it at $x=0$ or 1 . If it is at $x=0$, by the parabolic Hopf's lemma

$$
\left(u\left(x, t_{1}\right)-l(x)\right)_{x}>0 \text { at } x=0 .
$$

However, since $u\left(0, t_{1}\right)=l(0)$ and $l$ satisfies (1.2) at $x=0$, we have $u_{x}\left(0, t_{1}\right)-$ $l_{x}(0)=0$, contradiction holds. Therefore, $u$ must touch $l$ at $x=1$. At $x=1$, we have $u\left(1, t_{1}\right)=l(1)$. We have, by the parabolic Hopf's lemma,

$$
\begin{aligned}
0>u_{x}\left(1, t_{1}\right)-l_{x}(1) & =-\alpha_{2} u\left(1, t_{1}\right)+\beta_{2}-\left(a \alpha_{1}+\beta_{1}\right) \\
& =-\alpha_{2} l(1)+\beta_{2}-\left(a \alpha_{1}+\beta_{1}\right), \\
& =\beta_{2}-\left(1+\alpha_{2}\right) \beta_{1}>0,
\end{aligned}
$$

contradiction holds again. We conclude that $u(x, t)>l(x)$ for all time. As $u$ must be unbounded, by Lemma 3.3, $\min _{x} u(x, t) \rightarrow \infty$. When $\gamma<0$, a similar argument using (4.3) instead of (4.2) shows that $\max _{x} u(x, t) \rightarrow-\infty$ as $t \rightarrow \infty$.

For the case where $\alpha_{1}>0$ and $\alpha_{2}<0$, the function $v(x, t) \equiv u(1-x, t)$ satisfies (1.1) with boundary condition

$$
\begin{aligned}
& v_{x}(0, t)=\alpha_{2} v(0, t)-\beta_{2}, \\
& v_{x}(1, t)=-\alpha_{1} v(1, t)-\beta_{1} .
\end{aligned}
$$

Applying the previous arguments to $v$, we conclude that

$$
\begin{gathered}
\min _{x} u(x, t) \rightarrow \infty \quad \text { when } \gamma>0, \text { and } \\
\max _{x} u(x, t) \rightarrow-\infty \quad \text { when } \gamma<0
\end{gathered}
$$

when $\alpha_{1}>0$ and $\alpha_{2}<0$. We have settled the case for $\alpha$ in $\Gamma_{1}$.

Third, consider $\alpha$ in $D_{2}$. In this case $\delta$ is negative. For large $a>0$ (resp. $<0$ ), the function $w$ in $(4.1)$ becomes convex (resp. concave) and tends to $\infty$ (resp. $-\infty)$ as $a \rightarrow \infty$ (resp. $-\infty)$. As there is only one steady state in this case, for all sufficiently large $a$, the global solutions starting at $w$ will diverge to $\infty$ or $-\infty$ as $t \rightarrow \infty$ depending on whether $a$ is positive or negative by Lemmas 3.1 and 3.3 . 
To complete the proof it remains to exclude Case 4. To achieve this we assume such solution exists and draw a contradiction. Let $u$ be such a solution. By the strong maximum principle and Lemma 3.2, we have either $u(0, t) \rightarrow \infty$ and $u(1, t) \rightarrow-\infty$ or $u(0, t) \rightarrow-\infty$ and $u(1, t) \rightarrow \infty$. Replacing $u$ by $v(x, t)=u(1-x, t)$ if necessary, we may assume that $u(0, t) \rightarrow \infty$ and $u(1, t) \rightarrow-\infty$. By the mean value theorem $\min _{x} u_{x}(x, t) \rightarrow-\infty$ as $t \rightarrow \infty$. We can find a large, positive $t_{0}$ such that $\min _{x} u_{x}(x, t) \geq \min _{x} u_{x}\left(x, t_{0}\right)$ for all $t \leq t_{0}$. Since $u_{x}$ satisfies a linear parabolic equation, by the strong maximum principle either $(\mathrm{a}) \min _{x} u_{x}\left(x, t_{0}\right)=u_{x}\left(0, t_{0}\right)$ or $(\mathrm{b}) \min _{x} u_{x}\left(x, t_{0}\right)=u_{x}\left(1, t_{0}\right)$. We first show that (b) is impossible. For, suppose it holds. Consider in (4.3) the linear function $\tilde{l}$ which satisfies $\tilde{l}(1)=u\left(1, t_{0}\right)$ and $\tilde{l}_{x}(1)=u_{x}\left(1, t_{0}\right)$. From (b) we see that $\tilde{l}(x)>u\left(x, t_{0}\right)$ for all $x \in[0,1)$. As $u_{t}(1, t)<0$ for all $t \geq t_{0}$ (Lemma 3.2), $u$ can never touch $\tilde{l}$ again at $x=1$. In case there is a first time $t_{1}>t_{0} u$ touches $\tilde{l}$ again, it must occur at $x=0$. At this time, $u\left(0, t_{1}\right)=\tilde{l}(0)=a\left(1+\alpha_{2}\right)-\beta_{2}$, so by parabolic Hopf's lemma,

$$
\begin{aligned}
0<(\tilde{l}-u)_{x}(0, t) & =-a \alpha_{2}+\beta_{2}-\left(\alpha_{1} u\left(0, t_{1}\right)+\beta_{1}\right) \\
& =-a \delta+\left(1+\alpha_{1}\right) \beta_{2}-\beta_{1} .
\end{aligned}
$$

However, as $a=\tilde{l}(1)=u\left(1, t_{0}\right)$ is very negative and $\delta<0$ the term $-a \delta+$ $\left(1+\alpha_{1}\right) \beta_{2}-\beta_{1}$ is negative, contradiction holds. Therefore, $\min _{x} u_{x}$ must occur at $x=0$ at $t_{0}$. But then consider $l$ in (4.2) which satisfies $a=l(0)=u\left(0, t_{0}\right)$. It lies below $u\left(\cdot, t_{0}\right)$. As $u_{t}(0, t)>0$ for all $t \geq t_{0}, u$ and $l$ can only touch at a first time $t_{1}>t_{0}$ at $x=1$. However, then $u\left(1, t_{1}\right)=l(1)=a\left(1+\alpha_{1}\right)+\beta_{1}$,

$$
\begin{aligned}
0>(u-l)_{x}(1, t) & =-\alpha_{2} u\left(1, t_{1}\right)+\beta_{2}-\left(a \alpha_{1}+\beta_{1}\right) \\
& =-\delta a+\gamma .
\end{aligned}
$$

As $a$ is large and $\delta<0,-\delta a+\gamma>0$, contradiction holds. Therefore, $u>l$ for all time, and so the minimum of this solution tends to infinity. We have shown that there are no unbounded solutions which are unbounded from both sides.

We now come to $\alpha$ in $\Gamma_{2}$. In this case, the straight lines given by the graphs of $l$ in (4.2) and $\tilde{l}$ in (4.3) both have zeros approaching $-1 / \alpha_{1} \in(0,1)$ as $|a| \rightarrow \infty$. For a large $a>0, \max \{l, \tilde{l}\}$ is a convex function whose boundary values are very large and satisfies (1.2). We may smooth it out at their intersection to get a smooth convex function $u_{0}$. By solving (1.1) under (1.2) with this $u_{0}$ as initial function we obtain an unbounded solution which diverges to $\infty$ uniformly as $t \rightarrow \infty$. By smoothing out $\min \{l, \tilde{l}\}$ for a large, negative $a$, we obtain a concave solution which diverges to $-\infty$ uniformly.

When $\gamma=0$, the same arguments above involving (4.4) and (4.5) show that there is no global solution which tends to $\infty$ and $-\infty$ simultaneously.

When $\gamma \neq 0$, such solution exists by an indirect argument as shown below.

First, fix a convex $u_{10}$ and a concave $u_{20}, u_{10}>u_{20}$, so that the global solution $u_{1}$ and $u_{2}$ starting at $u_{10}$ and $u_{20}$ tend to $\infty$ and $-\infty$ respectively as $t \rightarrow \infty$. Let $u_{\lambda}$ be the solution starting at $(1-\lambda) u_{10}+\lambda u_{20}$. We claim that 
the non-empty set $\left\{\lambda \in[0,1]: \min u_{\lambda}(., t) \rightarrow \infty\right.$ as $\left.t \rightarrow \infty\right\}$ is open in $[0,1]$. For, if $\lambda_{1}$ is in this set, the corresponding solution $u_{\lambda_{1}}\left(x, t_{1}\right)>u_{10}$ at some $t_{1}$. By the continuity dependence of initial data, for all $\lambda$ near $\lambda_{1}$ the corresponding solution $u_{\lambda}$ will satisfy $u_{\lambda}\left(x, t_{1}\right)>u_{10}$. Since $u_{10}$ is convex, by the comparison principle and Lemma $3.1 u_{\lambda}(x, t) \rightarrow \infty$ as $t \rightarrow \infty$. In other words, $\lambda$ also belongs to this set. Similarly, one can also prove that the non-empty set $\left\{\lambda \in[0,1]: \max u_{\lambda}(., t) \rightarrow-\infty\right.$ as $\left.t \rightarrow \infty\right\}$ is open. Therefore, the number $\lambda^{*}=\sup \left\{\lambda \in[0,1]: \min u_{\lambda}(., t) \rightarrow \infty\right\}$ is strictly less than one and the solution $u_{\lambda^{*}}$ must tend to $\infty$ and $-\infty$ as $t \rightarrow \infty$ because there are no steady states.

Finally, let's consider $\alpha$ in $D_{3}$. In this case there is a unique steady state, and as in the previous case, there are large convex (resp. concave) solutions which tend to $\infty$ (resp. $-\infty$ ) as $t \rightarrow \infty$ respectively. We need to show the existence of solution which tends to $\infty$ and $-\infty$ simultaneously. The arguments in the last paragraph do produce a solution $u_{\lambda^{*}}$ which does not diverge to $\infty$ or $-\infty$ uniformly. However, since it may tend to the steady state, we cannot conclude there is a solution unbounded from both sides. To establish this fact, we need a more delicate argument.

Consider the functional

$$
I(u)=\frac{1}{2} \int_{0}^{1} u_{x}^{2} d x+\frac{\alpha_{2}}{2} u^{2}(1)+\frac{\alpha_{1}}{2} u^{2}(0)
$$

for any $\alpha$. By standard arguments, the minimization problem

$$
\lambda_{1}=\inf \left\{2 I(u):\|u\|_{L^{2}}=1, u \in H^{1}([0,1])\right\}
$$

has a minimizer $\varphi_{1}$ satisfying

$$
-\varphi_{1 x x}=\lambda_{1} \varphi_{1}
$$

with $\varphi_{1 x}(0)=\alpha_{1} \varphi_{1}(0)$ and $\varphi_{1 x}(1)=-\alpha_{2} \varphi_{1}(1)$. It is well-known that $\varphi_{1}$ does not change sign on $[0,1]$. We shall assume that it is positive in below. Also,

$$
\lambda_{2}=\inf \left\{2 I(u):\|u\|_{L^{2}}=1, u \in H^{1}([0,1]),\left\langle u, \varphi_{1}\right\rangle=0\right\}
$$

where

$$
\langle u, v\rangle=\int_{0}^{1} u_{x} v_{x} d x+\alpha_{2} u(1) v(1)+\alpha_{1} u(0) v(0)
$$

is attained at some $\varphi_{2}$ which satisfies $-\varphi_{2 x x}=\lambda_{2} \varphi_{2}$ with the same boundary conditions as $\varphi_{1}$. We have

$$
\begin{aligned}
& I\left(\varphi_{1}\right)=\frac{\lambda_{1}}{2} \\
& I\left(\varphi_{2}\right)=\frac{\lambda_{2}}{2}
\end{aligned}
$$

and, by $\left\langle\varphi_{1}, \varphi_{2}\right\rangle=0$,

$$
I\left((1-\lambda) \varphi_{1}+\lambda \varphi_{2}\right)=\frac{(1-\lambda)^{2} \lambda_{1}+\lambda^{2} \lambda_{2}}{2} .
$$

We claim that $\lambda_{1}$ and $\lambda_{2}$ are negative by explicitly considering the eigenvalue problem

$$
-\varphi_{x x}=\lambda \varphi
$$


subject to $\varphi_{x}(0)=\alpha_{1} \varphi(0)$ and $\varphi_{x}(1)=-\alpha_{2} \varphi(1)$. For any negative $\lambda$ the solution is of the form

$$
\varphi(x)=a e^{\mu x}+b e^{-\mu x}, \mu \equiv \sqrt{-\lambda} .
$$

In order to fulfill the boundary condition we require the linear system

$$
\left\{\begin{array}{l}
\left(\mu-\alpha_{1}\right) a-\left(\mu+\alpha_{1}\right) b=0 \\
\left(\mu+\alpha_{2}\right) e^{\mu} a+\left(-\mu+\alpha_{2}\right) e^{-\mu} b=0
\end{array}\right.
$$

to have non-trivial solution $(a, b)$, and this is true if and only if $\left(\mu-\alpha_{1}\right)$ $\left(-\mu+\alpha_{2}\right) e^{-\mu}+\left(\mu+\alpha_{2}\right)\left(\mu+\alpha_{1}\right) e^{\mu}=0$, that's,

$$
\alpha_{1} \alpha_{2}-\left(\alpha_{1}+\alpha_{2}\right) \mu+\mu^{2}=\left(\alpha_{1} \alpha_{2}+\left(\alpha_{1}+\alpha_{2}\right) \mu+\mu^{2}\right) e^{2 \mu} .
$$

Denote the left hand side of the above expression by $f(\mu)$ and the right hand side by $g(\mu)$. It is readily checked that $f(0)=g(0)$ and $g^{\prime}(0)>f^{\prime}(0)$, so the graph of $g$ lies above the graph of $f$ for $\mu$ near 0 . By solving the root for $f$, its discriminant shows that $f$ is positive in $[0, \infty)$ while $g$ vanishes at $-\alpha_{1}$ and $-\alpha_{2}$, which are both positive when $\alpha$ belongs to $D_{3}$. As $f / g$ tends to zero at $\infty$, the graphs of $f$ and $g$ must intersect at least twice. It follows that there are two choices $\mu_{1}$ and $\mu_{2}$ to make $\varphi$ a non-zero function. We conclude that the eigenvalue problem has at least two negative eigenvalues, in other words, $\lambda_{1}$ and $\lambda_{2}$ are negative.

Let's take $u_{01}=a \varphi_{1}+l_{s}$ and $u_{02}=a \varphi_{2}+l_{s}$ where $l_{s}$ is the unique steady state for (1.1) under (1.2). Both $u_{01}$ and $u_{02}$ satisfy (1.2). Consider the functional

$$
J(u)=\frac{1}{2} \int_{0}^{1} u_{x}^{2} d x+\frac{1}{2} \alpha_{2} u^{2}(1)+\frac{1}{2} \alpha_{1} u^{2}(0)-\beta_{2} u(1)+\beta_{1} u(0),
$$

which is decreasing along (1.1) and (1.2). Letting $\varphi_{\lambda}=(1-\lambda) a \varphi_{1}+\lambda a \varphi_{2}+l_{s}$, we have

$$
\begin{aligned}
J( & \left.(1-\lambda) u_{01}+\lambda u_{02}\right)=J\left(\varphi_{\lambda}\right) \\
= & I\left(\varphi_{\lambda}\right)-\beta_{2} \varphi_{\lambda}(1)+\beta_{1} \varphi_{\lambda}(0) \\
= & \frac{1}{2}\left[\lambda_{1}(1-\lambda)^{2}+\lambda_{2} \lambda^{2}\right] a^{2}+\frac{a}{2} \int_{0}^{1}\left[(1-\lambda) \lambda_{1} \varphi_{1}+\lambda \lambda_{2} \varphi_{2}\right] l_{s} d x \\
& \quad+\frac{\beta_{1}}{2} \varphi_{\lambda}(0)-\frac{\beta_{2}}{2} \varphi_{\lambda}(1) \\
< & 0
\end{aligned}
$$

provided $|a|$ is sufficiently large. Observing that $\varphi_{1}$ is a positive convex function, the solution $u_{1}$ starting at $a \varphi_{1}+l_{s}$ tends to $\infty$ (resp. $-\infty$ ) when $a$ is positive (resp. negative) and large. Suppose there are no unbounded solutions which tend to $\infty$ and $-\infty$ simultaneously. Consider the solution $u_{2}$ starting at $a \varphi_{2}+l_{s}, a>0$ large. Since it does not go unbounded from both sides by assumption nor tends to the steady state as $J\left(a \varphi_{2}+l_{s}\right)<0$ and $J\left(l_{s}\right)=0$, it must diverge to $\infty$ or $-\infty$. Let us assume it is the former. Now, we can argue as in the previous case by considering the sets $\left\{\lambda: \max u_{\lambda}(., t) \rightarrow-\infty\right.$ as $\left.t \rightarrow \infty\right\}$ and $\left\{\lambda: \min u_{\lambda}(., t) \rightarrow \infty\right.$ as $\left.t \rightarrow \infty\right\}$ where $u_{\lambda}$ is the solution starting at $(1-\lambda)$ 
$\left(-a \varphi_{1}\right)+\lambda a \varphi_{2}+l_{s}$ (denoted by $\left.\widetilde{\varphi}_{\lambda}\right)$ and $a$ is a fixed large positive number. Both sets are non-empty and open. As above, we have $J\left(\widetilde{\varphi}_{\lambda}\right)<0$. The inequality

$$
J\left(u_{\lambda}(x, t)\right) \leq J\left(\widetilde{\varphi}_{\lambda}\right)<0
$$

shows that $u_{\lambda}$ cannot converge to $l_{s}$, so it must be unbounded. It follows from Lemma 3.3 that $u_{\lambda^{*}}$, where $\lambda^{*}=\sup \left\{\lambda: u_{\lambda} \rightarrow-\infty\right.$ as $\left.t \rightarrow \infty\right\}$, must tend to $\infty$ and $-\infty$ simultaneously, contradiction holds. We conclude that there are solutions which are unbounded from both sides.

The proof of Theorem 1.1 is completed.

\section{Asymptotic behavior}

Now we prove Proposition 1.2. We shall assume $\alpha$ belongs to $\Gamma_{1}$ or $D_{2}$ where $\alpha_{1}<0$ and $\alpha_{2}>0$. The other case $\alpha_{1}>0$ and $\alpha_{2}<0$ can be treated similarly. We would like to show that for a convex global solution which tends to infinity, the curvature of its graph decays to zero in the part sitting inside the set $[\rho, 1-\rho] \times \mathbb{R}$ for every $\rho \in(0,1 / 2)$.

As the solution $u$ diverges to $\infty$, by Lemma 3.2 we may assume both $a(t) \equiv u(1, t)$ and $b(t) \equiv u(0, t)$ are increasing to $\infty$. By convexity, $u_{x}(x, t) \leq$ $u_{x}(1, t)=-\alpha_{2} u(1, t)+\beta_{2}$, so

$$
\max _{x \in[0,1]} u_{x}(x, t) \rightarrow-\infty, \quad \text { as } t \rightarrow \infty .
$$

For any $\rho \in(0,1 / 2)$ we fix $\rho^{\prime}$ and $\rho^{\prime \prime}$ with $0<\rho^{\prime \prime}<\rho^{\prime}<\rho$. From (5.1) we can find some $t_{1}$ such that

$$
u\left(\rho^{\prime}, t\right) \geq u(\rho, t)+3, \quad \forall t \geq t_{1} .
$$

From

$$
\begin{aligned}
\frac{d}{d t} \int_{\rho^{\prime \prime}}^{\rho^{\prime}} u d x & =\int_{\rho^{\prime \prime}}^{\rho^{\prime}} u_{t} d x \\
& =\arctan \left(u_{x}\left(\rho^{\prime}, t\right)\right)-\arctan \left(u_{x}\left(\rho^{\prime \prime}, t\right)\right)
\end{aligned}
$$

which tends to 0 as $t \rightarrow \infty$, for any given $\varepsilon$, there is some $t_{2} \geq t_{1}$ such that

$$
\left|\frac{d}{d t} \int_{\rho^{\prime \prime}}^{\rho^{\prime}} u d x\right| \leq \varepsilon, \quad \forall t \geq t_{2} .
$$

By the mean value theorem there exists $\rho(t) \in\left(\rho^{\prime \prime}, \rho^{\prime}\right)$ such that

$$
u(\rho(t), t)=\frac{\int_{\rho^{\prime \prime}}^{\rho^{\prime}} u(x, t) d x}{\rho^{\prime}-\rho^{\prime \prime}}, \quad \forall t \geq 0 .
$$


Therefore, for $t, t^{\prime} \in\left(t_{2}, \infty\right)$,

$$
\begin{aligned}
\left|u(\rho(t), t)-u\left(\rho\left(t^{\prime}\right), t^{\prime}\right)\right| & =\frac{1}{\rho^{\prime}-\rho^{\prime \prime}}\left|\int_{\rho^{\prime \prime}}^{\rho^{\prime}} u(x, t) d x-\int_{\rho^{\prime \prime}}^{\rho^{\prime}} u\left(x, t^{\prime}\right) d x\right| \\
& \leq \frac{\varepsilon\left|t-t^{\prime}\right|}{\rho^{\prime}-\rho^{\prime \prime}} .
\end{aligned}
$$

It follows that there is $t_{3} \geq t_{2}$ such that

$$
\left|u(\rho(t), t)-u\left(\rho\left(t^{\prime}\right), t^{\prime}\right)\right| \leq 1, \quad \forall t \in\left[t^{\prime}-1, t^{\prime}\right], \quad \forall t^{\prime} \geq t_{3} .
$$

Then, for $t \in\left[t^{\prime}-1, t^{\prime}\right]$ with $t^{\prime} \geq t_{3}$ and $x \in[\rho, 1-\rho]$,

$$
\begin{aligned}
u(\rho(t), t) & \geq u\left(\rho\left(t^{\prime}\right), t^{\prime}\right)-1 \\
& \geq u\left(\rho^{\prime}, t^{\prime}\right)-1(\text { as } u \text { is decreasing }) \\
& \geq u\left(\rho, t^{\prime}\right)+2(\text { by }(5.2)) \\
& \geq u\left(x, t^{\prime}\right)+2 .
\end{aligned}
$$

Since $b(t) \geq u(\rho(t), t)$, we have

$$
b(t)-1 \geq u\left(x, t^{\prime}\right)+1, \quad \forall t \in\left[t^{\prime}-1, t^{\prime}\right], \quad \forall t^{\prime} \geq t_{3},
$$

where $x \in[\rho, 1-\rho]$. By a similar consideration, there is some large time still denoted by $t_{3}$ such that

$$
\begin{aligned}
& a(t)+1 \leq u\left(x, t^{\prime}\right)-1, \\
& \quad \forall t \in\left[t^{\prime}-1, t^{\prime}\right], x \in[\rho, 1-\rho], \quad \forall t^{\prime} \geq t_{3} .
\end{aligned}
$$

We have shown that for any $x \in[\rho, 1-\rho]$,

$$
\begin{array}{r}
{\left[u\left(x, t^{\prime}\right)-1, u\left(x, t^{\prime}\right)+1\right] \subset(a(t), b(t)),} \\
\forall t \in\left[t^{\prime}-1, t^{\prime}\right], \forall t^{\prime} \geq t_{3} .
\end{array}
$$

This fact means that for any given $t^{\prime} \geq t_{3}$, during the time $\left[t^{\prime}-1, t^{\prime}\right]$, the inverse function $x=x(u, t)$ of $u(., t)$ is a graph over the interval $\left[u\left(x, t^{\prime}\right)-1, u\left(x, t^{\prime}\right)+1\right]$ with $x \in[\rho, 1-\rho]$. Moreover, by the geometric invariance of (1.1) it satisfies the same equation

$$
x_{t}=\frac{x_{u u}}{1+x_{u}^{2}},
$$

in the domain $\left(u\left(x, t^{\prime}\right)-1, u\left(x, t^{\prime}\right)+1\right) \times\left(t^{\prime}-1, t^{\prime}\right]$.

Now we claim that $\sup _{[\rho, 1-\rho]}|k(x, t)| \rightarrow 0$ as $t \rightarrow \infty$ where $k(x, t)$ is the curvature of the graph of $(x, u(x, t))$. For if not, there exist a sequence $\left\{\left(x_{j}, t_{j}\right)\right\}_{j=1}^{\infty}$ and a positive constant $C_{0}$ such that

$$
\left(x_{j}, t_{j}\right) \in[\rho, 1-\rho] \times\left(t_{3}, \infty\right), \quad t_{j} \rightarrow \infty,
$$

and

$$
k\left(x_{j}, t_{j}\right) \geq C_{0}>0 .
$$

On the other hand, observe that $\varphi_{j}(u, t) \equiv x\left(u+u\left(x_{j}, t_{j}\right), t+t_{j}\right)$ satisfies

$$
\varphi_{j t}=\frac{\varphi_{j u u}}{1+\varphi_{j u}^{2}},(u, t) \in(-1,1) \times(-1,0] .
$$


From (5.1) we see that $\varphi_{j u}$ is uniformly bounded in $(u, t) \in(-1,1) \times(-1,0]$. We may apply the interior estimate for quasi-linear parabolic equation (see, chapter 5 in [16]) to (5.4) in divergence form to conclude that the following estimate

$$
\left\|\varphi_{j}\right\|_{\tilde{C}^{1, \sigma}([-1 / 2,1 / 2] \times[-1 / 2,0])} \leq C_{1},
$$

holds for some constant $C_{1}$ and $\sigma \in(0,1)$. It implies that we can view (5.4) as a linear parabolic equation for $\varphi_{j}$ with Hölder coefficient $1 /\left(1+\varphi_{j u}^{2}\right)$. By parabolic Schauder theory the following higher order estimate

$$
\left\|\varphi_{j}\right\|_{\tilde{C}^{2, \sigma}([-1 / 4,1 / 4] \times[-1 / 4,0])} \leq C_{2},
$$

holds for some $C_{2}$. Consequently, there exist a subsequence $\varphi_{j_{k}}$ and a function $\varphi$ such that

$$
\varphi_{j_{k}}(u, 0) \rightarrow \varphi \text { in } C^{2}([-1 / 4,1 / 4]), \quad k \rightarrow \infty .
$$

By (5.1) we have

$$
\sup _{u \in[a(t), b(t)]}\left|x_{u}(u, t)\right| \rightarrow 0, \quad t \rightarrow \infty,
$$

which implies that $\sup _{u \in[-1 / 4,1 / 4]}\left|\left(\varphi_{j}\right)_{u}(u, 0)\right| \rightarrow 0$ as $j \rightarrow \infty$. So $\varphi_{u} \equiv 0$ and $\varphi_{u u} \equiv 0$. In other words, $\varphi_{j_{k} u u}(0,0) \rightarrow 0$ as $k \rightarrow \infty$. Noting that

$$
\varphi_{j_{k} u u}(0,0)=x_{u u}\left(u\left(x_{j_{k}}, t_{j_{k}}\right), t_{j_{k}}\right),
$$

we conclude that $k\left(x_{j_{k}}, t_{j_{k}}\right)=\varphi_{j_{k} u u}(0,0) /\left(1+\varphi_{j_{k} u}^{2}(0,0)\right)^{3 / 2} \rightarrow 0$ as $k \rightarrow \infty$, contradiction holds. Hence the curvature must decay to zero as time approaches infinity.

The proof of Proposition 1.2 is completed.

Next we prove Proposition 1.3. For any global solution which becomes unbounded from both sides we would like to establish the decay of the curvature of its graph in $[0,1] \times[-c, c]$ for any $c>0$. We know that such solution $u$ is possible only for $\Gamma_{2}$ or $D_{3}$ where both $\alpha_{1}$ and $\alpha_{2}$ are negative. As min $u(., t)$ and $\max u(., t)$ become unbounded, by the strong maximum principle either $u(0, t)$ tends to $\infty$ and $u(1, t)$ to $-\infty$ or the other way around. For definiteness let us assume it is the former case. In fact, the latter case can be reduced to the former by looking at $u(1-x, t)$.

First we argue that the solution $u(., t)$ becomes strictly decreasing after some time. To see this we observe that both $u(0, t)=\max u(., t)$ and $-u(1, t)=$ $-\min u(., t)$ become very large after some time, and the maximum and minimum are not attained away from the endpoints. Therefore, we can find some $t_{1}$ and constants $c_{1}<u\left(0, t_{1}\right)$ and $c_{2}>u\left(1, t_{1}\right)$ so that $c_{1}$ is greater than any interior maximal value of $u\left(., t_{1}\right)$ and $c_{2}$ is less than any interior minimal value of $u\left(., t_{1}\right)$. The horizontal lines $\left(x, c_{1}\right)$ and $\left(x, c_{2}\right)$ intersect the graph of $u\left(., t_{1}\right)$ at exactly one point transversally, and $u\left(., t_{1}\right)$ is strictly decreasing in the set $\left\{x: u\left(x, t_{1}\right)>c_{1}\right.$ or $\left.u\left(x, t_{1}\right)<c_{2}\right\}$. By Sturm comparison theorem (Angenent [3]), the graph of $u(., t)$ is strictly decreasing in the set $\{x: u(x, t)>$ $c_{1}$ or $\left.u(x, t)<c_{2}\right\}$ for all $t \geq t_{1}$. For the portion of the graph inside the rectangle $[0,1] \times\left[c_{2}, c_{1}\right]$ we can use the method of foliation to show that it 
eventually becomes strictly decreasing after some $t_{2} \geq t_{1}$, see Angenent [4] or Chou-Zhu [8] for a similar argument.

After showing that $u(x, t)$ becomes strictly decreasing for $t \geq t_{2}$, the inverse function $x(u, t)$ of $u(x, t)$ satisfies (5.3) in $[a(t), b(t)] \times\left[t_{2}, \infty\right)$ where $a(t)=u(1, t)$ and $b(t)=u(0, t)$ tend to $-\infty$ and $\infty$ respectively in a monotonic way. Since $x_{u}(a(t), t)$ and $x_{u}(b(t), t)$ both tend to zero as $t$ tends to $\infty$, without loss of generality we may assume the minimum of $x_{u}$ along the parabolic boundary of $(a(t), b(t)) \times\left[t_{2}, \infty\right)$ is attained at $t_{2}$. By applying the maximum principle to the equation satisfied by $x_{u}$, we have

$$
0>x_{u}(u, t) \geq-\delta_{1},
$$

where $1 / \delta_{1}=-\min u_{x}\left(., t_{2}\right)$. For any $a>0$, by applying the interior estimate to the quasi-linear equation (5.3) (expressed in divergence form) in the domain $[-a-2, a+2] \times\left[t_{3}, \infty\right)$, we find a constant $C_{1}$ such that

$$
\left\|x_{u}\right\|_{\tilde{C}^{\sigma}\left([-a-1, a+1] \times\left[t_{3}+1, \infty\right)\right)} \leq C_{1},
$$

see, chapter 5 in [16]. Here $t_{3} \geq t_{2}$ so that $[-a-2, a+2] \subset\left[a\left(t_{3}\right), b\left(t_{3}\right)\right]$. It follows that we can view (5.3) as linear parabolic equation with Hölder coefficient $1 /\left(1+x_{u}^{2}\right)$. By the parabolic Schauder theory, we readily obtain the higher order interior estimate, namely, there exists a constant $C_{2}$ (depending on $a$ ) such that

$$
\|x\|_{\tilde{C}^{3, \sigma}\left([-a, a] \times\left[t_{3}+2, \infty\right)\right)} \leq C_{2} .
$$

For any positive $c$, we now claim that $x_{u}$ decays to zero uniformly on $[-c, c]$ as $t$ tends to $\infty$. For if not, there is some positive $C_{3}$ such that

$$
x_{u}\left(u_{k}, t_{k}\right) \leq-C_{3},
$$

where $u_{k} \in[-c, c]$ and $t_{k} \rightarrow \infty$. Let $\varphi_{k}(u, t)=x_{u}\left(u+u_{k}, t+t_{k}\right)$. By the above estimate (5.5), we can extract a diagonal subsequence of $\left\{\varphi_{k}\right\}$ which $C^{2}$-converges compactly to a solution $\varphi$ of the equation

$$
\varphi_{t}=\left(\frac{\varphi_{u}}{1+\varphi^{2}}\right)_{u}, \quad(u, t) \in(-\infty, \infty) \times(-\infty, \infty) .
$$

In particular, $\varphi(0,0)=\lim _{k \rightarrow \infty} \varphi_{k}(0,0) \leq-C_{3}$. However, on the other hand, noting that $\varphi$ is uniformly bounded, we conclude from Liouville theorem (Moser [18]) that $\varphi$ is a constant $\varphi(0,0)$. As

$$
1>\left|\int_{-a}^{a} \varphi_{k}(u, t) d u\right| \rightarrow\left|\int_{-a}^{a} \varphi(u, t) d u\right|=2 a|\varphi(0,0)|,
$$

for any positive $a$ as $k \rightarrow \infty$, one must have $\varphi(0,0)=0$, contradiction holds. Therefore $x_{u}$ must tend to zero uniformly on each bounded interval of $u$. By combining with the higher order estimate (5.5), we conclude that $x_{u u}$ also tends to zero on any bounded interval, so does the curvature of the graph of $x(u, t)$.

We have completed the proof of Proposition 1.3. 


\section{Acknowledgments}

The authors are grateful to the referee whose comments have helped improve the presentation of this paper.

\section{References}

[1] Altschuler, S.J., Wu, L.F.: Convergence to translating solutions for a class of quasilinear parabolic boundary problems. Math. Ann. 295, 761-765 (1993)

[2] Altschuler, S.J., Wu, L.F.: Translating surfaces of the non-parametric mean curvature flow with prescribed contact angle. Calc. Var. 2, 101-111 (1994)

[3] Angenent, S.: The zero set of a solution of a parabolic equation. J. Reine Angew. Math. 390, 79-96 (1988)

[4] Angenent, S.: Parabolic equations for curves on surfaces II. Intersections, blow up and generalized solutions. Ann. Math. 133, 171-215 (1991)

[5] Brunovský, P., Poláčik, P., Sandstede, B.: Convergence in general periodic parabolic equations in one space dimension. Nonlinear Anal. 18, 209-215 (1992)

[6] Cao, F.: Geometric curve evolution and image processing. In: Lecture Notes in Mathematics, vol. 1805. Springer, Berlin (2003)

[7] Chern, H.-H., Guo, J.-S., Lo, C.-P.: The self-similar expanding curve for the curvature flow equation. Proc. Amer. Math. Soc. 131, 3191-3201 (2003)

[8] Chou, K.-S., Zhu, X.P.: Shortening complete plane curves. J. Differ. Geom. 50, 471-504 (1998)

[9] Chou, K.-S., Zhu, X.-P.: The Curve Shortening Problem. Chapman \& Hall/CRC, Boca Raton (2001)

[10] Gage, M., Hamilton, R.: The heat equation shrinking convex plane curves. J. Differ. Geom. 23, 69-96 (1986)

[11] Giga, Y.: Surface evolution equations - a level set method. In: Monographs in Mathematics, vol. 99. Birkhäuser Verlag, Basel (2006)

[12] Grayson, M.A.: The heat equation shrinks embedded plane curves to round points. J. Differ. Geom. 26, 285-314 (1987)

[13] Huisken, G.: Non-parametric mean curvature evolution with boundary conditions. J. Differ. Equ. 77, 369-378 (1989)

[14] Huisken, G.: A distance comparison principle for evolving curves. Asian J. Math. 2, 127-133 (1998)

[15] Kohsaka, Y.: Free boundary problem for quasilinear parabolic equation with fixed angle of contact to a boundary. Nonlinear Anal. 45, 865-894 (2001) 
[16] Ladyzhenskaya, O.A., Solonnikov, V.A., Uraltseva, N.N.: Linear and Quasilinear Equations of Parabolic Type. Academic Press, New York (1968)

[17] Lieberman, G.M.: Second Order Parabolic Differential Equations. World Scientific Publishing Inc., River Edge (1996)

[18] Moser, J.: A Harnack inequality for parabolic differential equations. Comm. Pure Appl. Math. 17, 101-134 (1964)

[19] Rubinstein, J., Sternberg, P., Keller, J.: Fast reaction, slow diffusion, and curve shortening. SIAM J. Appl. Math. 49, 116-133 (1989)

[20] Sapiro, G.: Geometric partial differential equations and image analysis. Cambridge University Press, Cambridge (2006)

[21] Stahl, A.: Regularity estimates for solutions to the mean curvature flow with a Neumann boundary condition. Calc. Var. 4, 385-407 (1996)

[22] Zelenjak, T.I.: Stabilization of solutions of boundary value problems for a secondorder parabolic equation with one space variable. Differ. Equ. 4, 34-45 (1968)

[23] Zhu, X.-P.: Lectures on mean curvature flows. In: Studies in Advanced Mathematics. American Mathematical Society, Providence \& International Press, Somerville (2002)

Kai-Seng Chou

Department of Mathematics

The Chinese University of Hong Kong

Shatin, Hong Kong

e-mail: kschou@math.cuhk.edu.hk

Xiao-Liu Wang

Department of Mathematics

Southeast University

No. 2 Sipailou

Nanjing 210096

People's Republic of China

e-mail: xlwang.seu@gmail.com

Received: 30 October 2010.

Accepted: 3 June 2011. 